\title{
MULTI-AGENT AVOIDANCE CONTROL USING AN M-MATRIX PROPERTY*
}

\author{
DUŠAN M. STIPANOVIĆ ${ }^{\dagger}$, SRIRAM SHANKARAN $^{\ddagger}$, AND CLAIRE J. TOMLIN T $^{\ddagger}$
}

\begin{abstract}
In this paper a generalization of avoidance control for multi-agent dynamic systems is presented. Strategies for avoidance control for multiple agents are obtained using individual Liapunov-type functions. The overall system avoidance conditions are guaranteed using a vector Liapunov-type function via an $M$-matrix property.
\end{abstract}

Key words. Avoidance control, $M$-matrices, Multi-agent systems.

AMS subject classifications. 15A99, 93C41, 93B52, 34H05, 49N30, 49N90.

1. Introduction. The problem of avoidance control for two independent agents has been introduced and extensively studied in a number of publications by Leitmann and his coworkers ([12]-[14], [5], and [6]). Avoidance control conditions guarantee that all the trajectories of a given dynamic system that start outside of the prescribed avoidance set in the state space, will never enter the set. These conditions are established using a Liapunov-type function.

In the past several years, a number of other tools for computing avoidance control conditions have been developed, generally based on computing reachable sets from the prescribed avoidance set. Methods include numerical computation of solutions to static Hamilton-Jacobi equations [1] and to techniques from viability theory and set valued analysis [4]. In other related work, we have developed a reachability computation method based on level set techniques [17] and viscosity solutions to Hamilton-Jacobi equations [7], using the ideas presented in [23]. In this previous work, we have represented a set as the zero sublevel set of an appropriate function, and the boundary of this set is propagated under the nonlinear dynamics using a validated numerical approximation of a time dependent Hamilton-Jacobi-Isaacs (HJI) partial differential equation (PDE) governing system dynamics [16].

In this paper, we generalize avoidance control conditions to the case of multiagent dynamic systems modeled as a class of nonlinear dynamic systems with a special decomposed structure ([21], [20]). The main novelty is that the results are established using a vector Liapunov-type function (vector Liapunov functions were independently introduced by Matrosov [15] and Bellman [2]) which is given as a positive linear combination of individual Liapunov-type functions that correspond to independent agents. Each individual function is dependent only on a subset of the state space variables, and strategies for avoidance control for each agent are thus defined using only these local state variables. Using a property of an $M$-matrix ([8], [3], [10]),

* Received by the editors 3 December 2004. Accepted for publication 5 May 2005. Handling Editor: Abraham Berman.

$\dagger$ Department of General Engineering and Coordinated Science Laboratory, University of Illinois at Urbana-Champaign, Urbana, IL 61801, USA (dusan@uiuc.edu).

$\ddagger$ Department of Aeronautics and Astronautics, Stanford University, Stanford, CA 94305, USA (ssriram@stanford.edu, tomlin@stanford.edu). 
conditions for avoidance control are established for the overall system, similar to the development of the connective stability results for interconnected systems ([21], [22], [18], and [19]).

2. $M$-matrices. $M$-matrices ([8], [3], [10]) are known to have many applications in modeling dynamic systems in Economics, Ecology, and Engineering [21]. Various properties of $M$-matrices are used in establishing stability results for dynamic systems in general (e.g., see [21] and [22], and the list of references reported therein). Since avoidance conditions may be linked to a stability type of result via Liapunovtype functions, $M$-matrices may be used to certify avoidance conditions. Avoidance conditions guarantee that all trajectories of a given dynamic system never enter the avoidance set which is a prescribed set in the state space. In this paper, the standard notation is used to denote the set of all square matrices of dimension $n$ with nonpositive off-diagonal elements as $\mathbf{Z}_{n}, \mathbf{Z}_{n} \subset \mathbb{R}^{n \times n}$. Then, the set of $M$-matrices, denoted as $\mathbf{M}_{n}, \mathbf{M}_{n} \subset \mathbf{Z}_{n}$, is defined as ([3], [10]):

Definition 2.1. A square $n$-dimensional matrix $A$ is said to be an $M$-matrix if $A \in \mathbf{Z}_{n}$ and if all eigenvalues of $A$ have positive real parts. The set $\mathbf{M}_{n}$ is a set of all $M$-matrices of dimension $n$.

Now, the following theorem formulates the property needed to establish avoidance conditions [3]:

Theorem 2.2. Let $A \in \mathbf{Z}_{n}$. Then, $A \in \mathbf{M}_{n}$ if and only if there exists a vector $d>0, d \in \mathbb{R}^{n}$, such that $A d>0$.

The linear programming test for the result in Theorem 2.2 is given by [18]:

Lemma 2.3. Let $A \in \mathbf{Z}_{n}$. Define the augmented matrix $\bar{A} \in \mathbb{R}^{n \times(n+1)}$ such that $\bar{A}=[A,-e]$, where $e=[1,1, \ldots, 1]^{T} \in \mathbb{R}^{n}$, and the augmented vector $\bar{d}=$ $\left[d_{1}, d_{2}, \ldots, d_{n}, d_{n+1}\right]^{T} \in \mathbb{R}^{n+1}$. Consider the following linear programming problem:

$$
\begin{aligned}
& \max d_{n+1} \\
& \text { subject to } \bar{A} \bar{d} \geq 0, \bar{d} \geq 0, d_{n+1} \leq 1
\end{aligned}
$$

Then, $A$ is an M-matrix if $d_{n+1}^{o}=\max d_{n+1}=1$.

3. Multi-Agent Avoidance Control. Let us consider a dynamic system described by the following:

$$
\dot{x}=f(x, u, w), \quad t \in \mathbf{T}=[0,+\infty), x(0) \in \mathbb{R}^{n},
$$

where $x \in \mathbb{R}^{n}$ is the state, $u \in \mathbb{R}^{m}$ is a known control input, and $w \in \mathbb{R}^{p}$ is an unknown input, or disturbance. Both the known and the unknown input are assumed to belong to a set of feedback strategies, that is, $u \in \mathcal{U}=\left\{\phi(\cdot): \mathbb{R}^{n} \rightarrow \mathbf{U}, \mathbf{U} \subset \mathbb{R}^{m}\right\}$ and $w \in \mathcal{W}=\left\{\psi(\cdot): \mathbb{R}^{n} \rightarrow \mathbf{W}, \mathbf{W} \subset \mathbb{R}^{p}\right\}$, and the function $f: \mathbb{R}^{n} \times \mathcal{U} \times \mathcal{W} \rightarrow \mathbb{R}^{n}$ is assumed to be Lipschitz continuous. Sets $\mathbf{U}$ and $\mathbf{W}$ are assumed to be compact. The input functions $t \rightarrow u(x(t))$ and $t \rightarrow w(x(t))$ are assumed to be measurable functions, and the trajectory of the system $t \rightarrow x(t)$ is an absolute continuous function such that equation (3.1) is satisfied almost everywhere. The set of input feedback strategies defines the admissible controls. 
The main idea of avoidance control is to develop an admissible control such that a particular set in the state space will be avoided for all possible unknown inputs, that is, disturbances. To be mathematically precise, we recall the following two definitions $[5]$ :

Definition 3.1. The dynamic system $\dot{x}=f(x, u(x), w(x))$ avoids $\Omega, \Omega \subset \mathbb{R}^{n}$, if and only if for each solution $x(t), t \in \mathbf{T}$, with $x(0) \notin \Omega$ the following is true: $x(t) \notin \Omega$ for all $t \in \mathbf{T}$.

DEFINITION 3.2. The set $\Omega$ is avoidable if there exists $u^{\circ} \in \mathcal{U}$ such that for any $w \in \mathcal{W}$ the system $\dot{x}=f(x, u(x), w(x))$ avoids $\Omega$.

In order to guarantee avoidance of the set $\Omega$, a safety region is constructed around the avoidance set such that there exists a Liapunov-type function which is nonincreasing in the safety region and becomes infinitely large on the boundary of the avoidance set. This is expressed in the following definition of the safety zone and a theorem involving a Liapunov-type function [5]:

Definition 3.3. $\Gamma$ is a safety zone for $\Omega$ if and only if $\Gamma \cap \Omega=\{\emptyset\}$ and $\partial \Omega \subset \operatorname{int}\{\Gamma \cup \Omega\}$.

TheOREM 3.4. Assume that there exist a function $u^{o}, u^{o} \in \mathcal{U}$ and a continuously differentiable function $v: \mathcal{O} \rightarrow \mathbb{R}$, where $\mathcal{O}$ is an open subset of $\mathbb{R}^{n}$ such that: (a) $\Gamma \subset \mathcal{O}$; (b) if $\hat{x} \in \partial \Omega$ then $\lim _{x \rightarrow \hat{x}} v(x)=\infty$; (c) for each $w \in \mathcal{W}$ and all $x \in \Gamma$, $\left.\frac{d v}{d t}\right|_{\dot{x}=f(x, u, w)}=\frac{\partial v}{\partial x} f(x, u, w) \leq 0$. Then, the set $\Omega$ is avoidable.

We use the standard notation $\partial \Omega$ and int $\{\Omega\}$ to denote the boundary and the interior of $\Omega$, respectively.

Notice that the preceding three definitions and theorem have been formulated for two agents only. Since our goal is to present a generalization of these results for a group of agents, let us assume that there are $N$-agents with independent control inputs $u_{i}, u_{i} \in \mathbb{R}^{m_{i}}$, such that $\sum_{i=1}^{N} m_{i}=m$. These are the agents with intentions to avoid the avoidance set. The other group of agents are the ones that would want to steer the system into the avoidance set. They are grouped in vector $w$ and need not be treated as individuals. From this point we will engage in the more formal description of the subsystem state spaces and we refer to the example at the end of the paper for the physical motivation of our approach. The avoidance set $\Omega$ is given in terms of a partition of the overall state as $x=\left[x^{1}, \ldots, x^{k}, \ldots x^{P}\right]$ with $x^{k} \in \mathcal{O}^{k}, \sum_{i=1}^{P} n^{k}=n$. $\mathcal{O}^{k}$ is an open subset of $\mathbb{R}^{n^{k}}$ for all $k \in \mathbf{P}=\{1, \ldots, P\}$. The avoidance set in the $x^{k}$ space is denoted as $\Omega^{k} \subset \mathcal{O}^{k}$ and is assumed to be an open set. Then, the overall avoidance set can be expressed using the extended sets $\widetilde{\Omega}^{k}=\mathcal{O}^{1} \times \ldots \times \underbrace{\Omega^{k}}_{k-\text { th place }} \times \ldots \times \mathcal{O}^{P}$, as $\Omega=\bigcup_{k=1}^{P} \widetilde{\Omega}^{k} \subset \mathcal{O}=\underset{k \in \mathbf{P}}{\times} \mathcal{O}^{k}$. The standard Cartesian product is denoted by $\times$. Notice that $\Omega$ and $\mathcal{O}$ are open sets since both the Cartesian product and the union of a finite number of open sets are open [11], [9].

To each agent we associate the subsystem state $x_{i}$ which is defined as a collection 
of $x^{k}$ 's, that is, $x_{i}=\underset{k \in \mathcal{C}_{i}}{\times} x^{k} \in \mathcal{O}_{i}=\underset{k \in \mathcal{C}_{i}}{\times} \mathcal{O}^{k}$, where the set $\mathcal{C}_{i} \subset \mathbf{P}$ is the set of indices that describes the subsystem $i$. The feedback structure is still assumed except now each independent agent forms its feedback control using only a portion of the state. Thus, the input sets for the agents are more specific: $u_{i} \in \mathcal{U}_{i}=\left\{\phi_{i}(\cdot): \mathcal{O}_{i} \rightarrow\right.$ $\left.\mathbf{U}_{i}, \mathbf{U}_{i} \subset \mathbb{R}^{m_{i}}\right\}$, where $\mathbf{U}_{i}$ for all $i \in \mathbf{N}$ are assumed to be compact. The goal for these independent agents stays the same, that is, to avoid the set $\Omega$. Additionally, we assume a particular structure for the system that is expressed in terms of its dynamic model. This structure is represented as a decomposition of the dynamic system [20]:

$$
\dot{x}_{i}=f_{i}(x, u, w)=g_{i}\left(x_{i}, u_{i}\right)+h_{i}(x, u, w), i \in \mathbf{N}=\{1,2, \ldots, N\},
$$

where $g_{i}: \mathcal{O}_{i} \times \mathcal{U}_{i} \rightarrow \mathbb{R}^{n_{i}}$ is a Lipschitz continuous function that represents subsystem dynamics (the one corresponding to the independent agent $i$ ), and $h_{i}: \mathcal{O} \times \mathcal{U} \times$ $\mathcal{W} \rightarrow \mathbb{R}^{n_{i}}$ is a Lipschitz continuous function that represents interconnections between subsystems, for all $i, i \in \mathbf{N}$. Notice that, by their construction, the subsystem states $x_{i} \in \mathbb{R}^{n_{i}}, i \in \mathbf{N}$ are not necessarily disjoint, that is, they may overlap $\left(x_{i} \cap x_{j} \neq\{\emptyset\}\right.$ for some $i, j \in \mathbf{N}$ ). This means that the same applies for $f_{i}$ and $f_{j}$. Also, we assume that $\sum_{i=1}^{N} n_{i} \geq n$, in general.

If $\Gamma^{k}$ is a safety region for $\Omega^{k}$ then the safety region for $x_{i}$, denoted as $\Gamma_{i}$, is given via the extended sets $\widehat{\Gamma}^{k}$, that are computed as the Cartesian products of $\Gamma^{k}$ and $\mathcal{O}^{j} \backslash \Omega^{j}$ for all $j \in \mathcal{C}_{i} \backslash\{k\}$, as $\Gamma_{i}=\bigcup_{k \in \mathcal{C}_{i}} \widehat{\Gamma}^{k}$. Notice that, by construction, $\Gamma_{i}$ is an open set that satisfies $\Gamma_{i} \subset \mathcal{O}_{i}$. Similarly, we can compute $\Gamma=\bigcup_{k \in \mathbf{P}} \widetilde{\Gamma}^{k}$, where $\widetilde{\Gamma}^{k}=\left\{\mathcal{O}^{1} \backslash \Omega^{1}\right\} \times \ldots \times \underbrace{\Gamma^{k}}_{k-\text { th place }} \times \ldots \times\left\{\mathcal{O}^{P} \backslash \Omega^{P}\right\}$, for all $k \in \mathbf{P}$.

With each subsystem state $x_{i}$ we associate a positive and continuously differentiable function $v_{i}\left(x_{i}\right)$. Our goal is to construct a function that will be infinitely large on each $\partial \Omega^{k}, k \in \mathcal{C}_{i}$. One possible solution (the one we propose in this paper and is certainly not unique) is to set

$$
v_{i}\left(x_{i}\right)=\sum_{k \in \mathcal{C}_{i}} v_{i k}\left(x^{k}\right)
$$

where $v_{i k}: \mathcal{O}^{k} \rightarrow \mathbb{R}_{+}\left(\mathbb{R}_{+}\right.$denotes the set of all nonnegative real numbers) with the additional property

$$
\lim _{x^{k} \rightarrow \hat{x}^{k}} v_{i k}\left(x^{k}\right)=\infty \text { if } \hat{x}^{k} \in \partial \Omega^{k} \text { and } k \in \mathcal{C}_{i} .
$$

Next, the Hamiltonian with respect to the subsystem dynamics is defined as [20]

$$
H_{g_{i}}\left(x_{i}, \frac{\partial v_{i}}{\partial x_{i}}\right)=\min _{u_{i} \in \mathbf{U}_{i}} \frac{\partial v_{i}}{\partial x_{i}} g_{i}\left(x_{i}, u_{i}\right)
$$


and the subsystem optimal control laws are obtained by the following:

$$
u_{i}^{o}\left(x_{i}\right)=\underset{u_{i} \in \mathbf{U}_{i}}{\arg \min }\left\{\frac{\partial v_{i}}{\partial x_{i}} g_{i}\left(x_{i}, u_{i}\right)\right\}, i \in \mathbf{N} .
$$

Notice that from (3.6) it follows that $u_{i}^{o} \in \mathcal{U}_{i}$.

Additionally, we assume that there exist positive numbers $\alpha_{i}$ and positive functions $\mu_{i}(\cdot): \mathcal{O}_{i} \rightarrow \mathbb{R}_{+}$such that

$$
H_{g_{i}}\left(x_{i}, \frac{\partial v_{i}}{\partial x_{i}}\right) \leq-\alpha_{i} \mu_{i}\left(x_{i}\right), \text { for all } x_{i} \in \Gamma_{i} .
$$

Similarly, let us assume that there exist nonnegative numbers $\alpha_{i j}$ such that the constraints on the interconnections, that depend on the unknown control inputs $w$, are given by

$$
\max _{w \in \mathbf{W}} \frac{\partial v_{i}}{\partial x_{i}} h_{i}\left(x, u^{o}(x), w\right) \leq \sum_{j=1}^{N} \alpha_{i j} \mu_{j}\left(x_{j}\right), \text { for all } x \in \Gamma .
$$

Notice again that the result of the maximization in (3.7) implies that the maximizing $w^{o}$ belongs to $\mathcal{W}$ since it is a function of the state only.

In order to establish the avoidance result for the overall system we assume a vector Liapunov-type function of the form [21]:

$$
v(x)=\sum_{i=1}^{N} d_{i} v_{i}\left(x_{i}\right)
$$

where the $d_{i}$ 's are all positive numbers (implying $v: \mathcal{O} \rightarrow \mathbb{R}_{+}$), and compute its time derivative along solutions of (3.2)

$$
\left.\frac{d v(x)}{d t}\right|_{(3.2)}=\sum_{i=1}^{N} d_{i} \frac{d v_{i}\left(x_{i}\right)}{d t}=\sum_{i=1}^{N} d_{i} \frac{\partial v_{i}}{\partial x_{i}} g_{i}\left(x_{i}, u_{i}\right)+\sum_{i=1}^{N} d_{i} \frac{\partial v_{i}}{\partial x_{i}} h_{i}(x, u, w)
$$

We follow the standard procedure [22], and define the $N \times N$ test matrix $Q=\left(q_{i j}\right)$ as

$$
q_{i j}= \begin{cases}\alpha_{i}-\alpha_{i i}, & i=j \\ -\alpha_{i j}, & i \neq j .\end{cases}
$$

Theorem 3.5. Let $Q$ be an $N \times N$ matrix as defined in (3.10). If $Q \in \mathbf{M}_{N}$ and $\mathbf{P} \subseteq \bigcup_{i=1}^{N} \mathcal{C}_{i}$ then the set $\Omega=\bigcup_{k=1}^{P} \widetilde{\Omega}^{k}$ is avoidable for the system (3.2) with the subsystem control strategies defined in (3.6). 
Proof. First, we establish part (a) of Theorem 3.4 by observing that if $\Gamma^{k}$ is a safety region for $\Omega^{k}$ then $\Gamma^{k} \subset \mathcal{O}^{k}$ which implies $\widetilde{\Gamma}^{k}=\left\{\mathcal{O}^{1} \backslash \Omega^{1}\right\} \times \ldots \times \underbrace{\Gamma^{k}}_{k-\text { th place }} \times \ldots \times$ $\left\{\mathcal{O}^{P} \backslash \Omega^{P}\right\} \subset \mathcal{O}$ for all $k \in \mathbf{P}$. The last set of inclusions implies $\Gamma=\bigcup_{k=1}^{P} \widetilde{\Gamma}^{k} \subset \mathcal{O}$.

Notice that the condition $\mathbf{P} \subseteq \bigcup_{i=1}^{N} \mathcal{C}_{i}$ implies that for any $k \in \mathbf{P}$ there exist $i \in \mathbf{N}$ and a function $v_{i k}(\cdot)$ such that (3.4) is satisfied. Part (b) follows directly from the the fact that the crossing of the boundary of any $\Omega^{k}$ implies existence of a subset of states $x^{k}$ such that $x^{k} \in \partial \Omega^{k}$, equations (3.3), (3.4), (3.8), and the fact that all Liapunov-type functions are nonnegative.

From equations (3.8) and (3.9) it follows that for $u_{i}^{o}\left(x_{i}\right), i \in \mathbf{N}$, as defined in equation (3.6) we get

$$
\left.\frac{d v(x)}{d t}\right|_{(3.2)}=\sum_{i=1}^{N} d_{i} H_{g_{i}}\left(x_{i}, \frac{\partial v_{i}}{\partial x_{i}}\right)+\sum_{i=1}^{N} d_{i} \frac{\partial v_{i}}{\partial x_{i}} h_{i}\left(x, u^{o}(x), w\right) \leq-d^{T} Q \mu(x)
$$

for all $x \in \Gamma$ with $\mu(x)=\left[\mu_{1}\left(x_{1}\right), \ldots, \mu_{N}\left(x_{N}\right)\right]^{T}$. In the above inequality we used the following property of the maximum operator

$$
\max _{w \in \mathbf{W}} \sum_{i=1}^{N} \frac{\partial v_{i}}{\partial x_{i}} h_{i}\left(x, u^{o}(x), w\right) \leq \sum_{i=1}^{N} \max _{w \in \mathbf{W}} \frac{\partial v_{i}}{\partial x_{i}} h_{i}\left(x, u^{o}(x), w\right) .
$$

From Definition 2.1 it follows that $Q$ is an $M$-matrix if and only if $Q^{T}$ is an $M$ matrix. Then, Theorem 2.2 implies that there exists a positive vector $d$ such that $Q^{T} d=c>0$. Thus, $\left.\frac{d v(x)}{d t}\right|_{(3.2)} \leq-c^{T} \mu(x) \leq 0$ for all $x \in \Gamma$ since $\mu(x)$ is a vector of nonnegative elements. Thus, part (c) is also established and the set $\Omega$ is avoidable via conditions of Theorem 3.4. $\square$

CoRollary 3.6. If $Q$ satisfies linear programming conditions of Lemma 2.3 and $\mathbf{P} \subseteq \bigcup_{i=1}^{N} \mathcal{C}_{i}$ then the system (3.2) avoids set $\Omega=\bigcup_{k=1}^{P} \widetilde{\Omega}^{k}$.

To illustrate the results presented in this paper we present the following example:

EXAMPLE 3.7. Let us consider a collision-avoidance problem with three vehicles such that the planar motion of the $i$-th vehicle, is described by the following noninertial kinematics model:

$$
\dot{z}_{i}=u_{i}
$$

where the state vector $z_{i} \in \mathbb{R}^{2}$ denotes rectangular coordinates in the plane, and $u_{i} \in \mathbb{R}^{2}$ is the control input representing velocity of the $i$-th vehicle. 
Since we are interested in controlling relative distances between the vehicles, using (3.11), we define

$$
\begin{aligned}
& x^{1}=z_{1}-z_{2} \Rightarrow \dot{x}^{1}=u_{1}-u_{2}, \\
& x^{2}=z_{1}-z_{3} \Rightarrow \dot{x}^{2}=u_{1}-u_{3}, \\
& x^{3}=z_{2}-z_{3} \Rightarrow \dot{x}^{3}=u_{2}-u_{3} .
\end{aligned}
$$

We assume that vehicles 1 and 2 are the ones that want to avoid the collision and that vehicle 3 is the one whose behavior we do not know and thus treat it as a disturbance. The domains for each of the $x^{i}$ states are given as $\mathcal{O}^{i}=\left\{x^{i}:\left\|x^{i}\right\| \leq R, R>0\right\}$, where $R$ is assumed to be sufficiently large. According to the appearance of their control inputs, with the vehicles 1 and 2 we associate subsystems states as $x_{1}=\underset{k \in\{1,2\}}{\times} x^{k}=$ $\left[x^{1}, x^{2}\right]$ and $x_{2}=\underset{k \in\{1,3\}}{\times} x^{k}=\left[x^{1}, x^{3}\right]$. For each $x^{i}$ we choose the corresponding avoidance set to be $\Omega^{k}=\left\{x^{k}:\left\|x^{k}\right\|<r_{1}\right\}$ and the Liapunov-type function of the form $v_{i k}\left(x^{k}\right)=\left(\left\|x^{k}\right\|-r_{1}\right)^{-2}$ for $i \in\{1,2\}$ and $k \in\{1,2,3\}$, where $r_{1}$ is a positive number determining the size of the avoidance set. Notice that the Liapunovtype functions satisfy (3.4). The safety regions may be chosen as "rings" around the avoidance regions such as $\Gamma^{k}=\left\{x^{k}: r_{1}<\left\|x^{k}\right\|<r_{2}\right\}$, where $R \gg r_{2}>r_{1}$. As an example of the bounding procedure, we choose the first subsystem, that is, $x_{1}=\left[x^{1}, x^{2}\right]$ and its corresponding dynamics as

$$
\dot{x}_{1}=\left[\begin{array}{c}
I_{2} \\
I_{2}
\end{array}\right] u_{1}-\left[\begin{array}{c}
I_{2} \\
0_{2}
\end{array}\right] u_{2}-\left[\begin{array}{c}
0_{2} \\
I_{2}
\end{array}\right] w
$$

where $I_{2}, 0_{2} \in \mathbb{R}^{2 \times 2}$ denote the identity and the zero matrix, respectively.

For the simplicity of the presentation we assume that $u_{i} \in \mathcal{U}_{i}=\left\{\phi_{i}(\cdot): \mathcal{O}_{i} \rightarrow \mathbf{U}_{i}\right\}$ with $\mathbf{U}_{i}=\left\{u_{i}:\left\|u_{i}\right\| \leq \nu_{i}, \nu_{i}>0\right\}, i \in\{1,2\}$, where $\|\cdot\|$ represents the Euclidean norm. For the third vehicle we assume that $w \equiv u_{3} \in \mathcal{W}=\{\psi(\cdot): \mathcal{O} \rightarrow \mathbf{W}, \mathbf{W} \subset$ $\left.\mathbb{R}^{2}\right\}$, where $\mathbf{W}$ is a compact set in $\mathbb{R}^{2}$. Notice that $g_{1}\left(x_{1}, u_{1}\right)=\left[I_{2}, I_{2}\right]^{T} u_{1}$ and using (3.6) we compute

$$
u_{1}^{o}\left(x_{1}\right)=\underset{u_{1} \in \mathbf{U}_{1}}{\arg \min }\left\{\frac{\partial v_{1}}{\partial x_{1}}\left[I_{2}, I_{2}\right]^{T} u_{1}\right\}=-\nu_{1}\left[I_{2}, I_{2}\right]{\frac{\partial v_{1}^{T}}{\partial x_{1}}}^{T} /\left\|\left[I_{2}, I_{2}\right] \frac{\partial v_{1}}{\partial x_{1}}\right\|
$$

and by noting that $g_{2}\left(x_{2}, u_{2}\right)=\left[-I_{2}, I_{2}\right]^{T} u_{2}$, similarly we obtain

$$
(3.13) u_{2}^{o}\left(x_{2}\right)=\underset{u_{2} \in \mathbf{U}_{2}}{\arg \min }\left\{\frac{\partial v_{2}}{\partial x_{2}}\left[I_{2}, I_{2}\right]^{T} u_{2}\right\}=-\nu_{2}\left[-I_{2}, I_{2}\right]{\frac{\partial v_{2}}{\partial x_{2}}}^{T} /\left\|\left[-I_{2}, I_{2}\right]{\frac{\partial v_{2}}{\partial x_{2}}}^{T}\right\| \text {, }
$$

where (again for the simplicity of the presentation) we assume that $u_{i}^{o}$ 's are properly defined, that is, the denominators are different from zero. From equations (3.5),(3.6), (3.12), and (3.13), we obtain

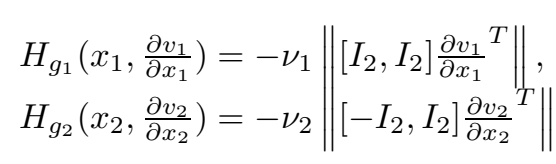


and set

$$
\alpha_{i} \equiv \nu_{i}, \mu_{i}\left(x_{i}\right) \equiv-\nu_{i}^{-1} H_{g_{i}}\left(x_{i}, \frac{\partial v_{i}}{\partial x_{i}}\right), i \in\{1,2\} .
$$

If there are coefficients $\alpha_{i j}$ in (3.8) such that that the matrix $Q$ in (3.10) is an $M$ matrix then there is a guarantee that there will be no collisions between the agents. One of the avenues for the future research would be to find the mathematical relationship between $r_{1}, r_{2}, \nu_{1}, \nu_{2}$, and $\mathbf{W}$ such that $Q$ is an $M$-matrix.

4. Conclusions. In this paper a solution for the multi-player avoidance control problem is presented. Possible extensions for this work are numerous. One direction is to study refinement of the bounds on the unknown interconnections by considering special structures where the individual treatment of the agents with strategies that would steer the system trajectories into the avoidance set would be of interest. The other direction is to consider the problem of finding the correlation between the size of the unknown perturbations that represent disturbances and the size of the safety regions where the safety regions would be given as level sets of a vector Liapunov-type function. Any result that would link the size of the interconnections and the size of the safety regions would be relevant in the study of the safety verification of dynamic systems.

Acknowledgment. This research is supported by the DOD Multidisciplinary University Research Initiative (MURI) program "CoMotion", administered by the Office of Naval Research under Grant N00014-02-1-0720.

\section{REFERENCES}

[1] M. Bardi and I. Capuzzo-Dolcetta. Optimal Control and Viscosity Solutions of HamiltonJacobi-Bellman Equations. Birkhäuser, Boston, 1997.

[2] R. Bellman. Vector Lyapunov functions. SIAM Journal of Control, 1:32-34, 1962.

[3] A. Berman and R.J. Plemmons. Nonnegative Matrices in Mathematical Sciences. Academic Press, New York, NY, 1979.

[4] P. Cardaliaguet, M. Quincampoix, and P. Saint-Pierre. Set-valued numerical analysis for optimal control and differential games, in Stochastic and Differential Games: Theory and Numerical Methods, (M. Bardi,T. Parthasarathy, and T. E. S. Raghavan, eds.), vol. 4 of Annals of International Society of Dynamic Games, Birkhäuser, 1999.

[5] M. Corless, G. Leitmann, and J. Skowronski. Adaptive control for avoidance or evasion in an uncertain environment. Computers \& Mathematics with Applications, 13:1-11, 1987.

[6] M. Corless and G. Leitmann. Adaptive controllers for avoidance or evasion in an uncertain environment: Some examples. Computers \& Mathematics with Applications, 18:161-170, 1989.

[7] L.C. Evans and P.E. Souganidis. Differential games and representation formulas for solutions of Hamilton-Jacobi-Isaacs equations. Indiana University Mathematics Journal, 33:773-797, 1984.

[8] M. Fiedler and V. Ptàk. On matrices with non-positive off-diagonal elements and positive principal minors. Czechoslovakian Mathematical Journal, 12:382-400, 1962.

[9] P.R. Halmos. Measure Theory. Springer-Verlag, New York, NY, 1974.

[10] R.A. Horn and C.R. Johnson. Topics in Matrix Analysis. Cambridge University Press, Cambridge, UK, 1991.

[11] A.N. Kolmogorov and S.V. Fomin. Introductory Real Analysis. Dover Publications, New York, NY, 1975. 
[12] G. Leitmann and J. Skowronski. Avoidance control. Journal of Optimization Theory and Applications, 23:581-591, 1977.

[13] G. Leitmann. Guaranteed avoidance strategies. Journal of Optimization Theory and Applications, 32:569-576, 1980 .

[14] G. Leitmann and J. Skowronski. A note on avoidance control. Optimal Control Applications G3 Methods, 4:335-342, 1983.

[15] V.M. Matrosov. On the theory of stability of motion. Prikladnia Matematika i Mekhanika, 26:992-1000, 1962.

[16] I. Mitchell, A.M. Bayen, and C.J. Tomlin. Computing reachable sets for continuous dynamic games using level set methods. IEEE Transactions on Automatic Control. To appear.

[17] S. Osher and J.A. Sethian. Fronts propagating with curvature-dependent speed: Algorithms based on Hamilton-Jacobi formulations. Journal of Computational Physics, 79:12-49, 1988.

[18] D.M. Stipanović and D.D. Šiljak. Stability of polytopic systems via convex $M$-matrices and parameter-dependent Liapunov functions. Nonlinear Analysis, 40:589-609, 2000.

[19] D.M. Stipanović and D.D. Siljak. Connective stability of discontinuous dynamic systems. Journal of Optimization Theory and Applications, 115:711-726, 2002.

[20] D.M. Stipanović, I. Hwang, and C.J. Tomlin. Computation of an over-approximation of the backward reachable set using subsystem level set functions. Dynamics of Continuous, Discrete and Impulsive Systems, Series A: Mathematical Analysis, 11:399-411, 2004.

[21] D.D. Siljak. Large-scale Dynamic Systems: Stability and Structure. North-Holland, New York, 1978.

[22] D.D. Šiljak. Decentralized Control of Complex Systems. Academic Press, Boston, 1991.

[23] C.J. Tomlin, J. Lygeros, and S. Sastry. A game theoretic approach to controller design for hybrid systems. Proceedings of the IEEE, 88:949-970, July 2000. 